\title{
Déficit de vitamina $D$ en hombres y mujeres con deseo sexual hipoactivo: Estudio de prevalencia
}

Franklin José Espitia de la Hoz ${ }^{\text {a }}$

\author{
${ }^{a}$ Médico, Especialista en Ginecología y Obstetricia, Sexólogo, Clínica Sexológica Hathor y Clínica La Sagrada Familia. \\ ORCID: https://orcid.org/0000-0002-4581-9680, espitiafranklin@hotmail.com \\ DOI: https://doi.org/10.22517/25395203.23251
}

\section{Resumen}

Objetivo: Establecer la prevalencia del déficit de vitamina $\mathrm{D}$ en hombres y mujeres con deseo sexual hipoactivo.

Materiales y métodos: Estudio de corte transversal en el período 2011-2017 en Armenia, Colombia en una muestra de 107 participantes. Se incluyeron hombres y mujeres con diagnóstico en la consulta externa, de una clínica privada de carácter universitario de referencia, de deseo sexual hipoactivo (DSH). Se les realizó cuantificación de 25-hidroxivitamina $\mathrm{D}$ [25(OH)D] (calcidiol) y se consideró que en la historia clínica tuvieran diligenciados los cuestionarios: Decreased Sexual Desire Screener (DSDS) o Prueba del Deseo Sexual Inhibido (PDSI), en las mujeres, y el cuestionario Sexual desire inventory (SDI) en los hombres. Muestreo no probabilístico por conveniencia. Se aplicó estadística descriptiva. Los resultados se exponen de manera agrupada para el total de la población.

Resultados: La prevalencia del déficit de vitamina D fue del $63.55 \%$ (34.57\% en hombres y $28.97 \%$ en mujeres). Los niveles de vitamina $\mathrm{D}$ fueron inferiores en los hombres respecto a las mujeres, ( 34.57 vs. 41.36 ; $\mathrm{p}<0.001)$, con mayor porcentaje de deficiencia ( $21.49 \mathrm{vs}$. $14.95 \% \mathrm{p}<0.007)$ y de insuficiencia $(16.82$ vs. $10.28 \%$, $\mathrm{p}<0.001)$.

Conclusiones: Es considerablemente alta la prevalencia $(63.55 \%)$ del déficit de vitamina $\mathrm{D}$, en hombres y mujeres con deseo sexual hipoactivo. Se hace necesaria la cuantificación de sus niveles a fin de establecer la suplencia como parte del tratamiento.

Palabras clave: Deficiencia de Vitamina D, Disfunciones sexuales fisiológicas, Salud Sexual, vitamina D.

\section{Vitamin D deficiency in men and women with hypoactive sexual desire disorder. Prevalence study}

\begin{abstract}
Objective: To establish the prevalence of vitamin D deficiency in men and women with hypoactive sexual desire disorder (HSDD).
\end{abstract}

Materials and methods: This cross-sectional study was conducted between 2011 and 2017, in Armenia, Colombia to a group of 107 participants (men and women) that were diagnosed with hypoactive sexual desire disorder (HSDD) in the outpatient department of a private University Referral Clinic. The participants underwent quantification of 25-hydroxyvitamin D [25 $(\mathrm{OH}) \mathrm{D}]$ (calcidiol) and it was confirmed if the medical records of the participants had completed some specific questionnaires. The Decreased Sexual Desire Screener (DSDS) or Test of Inhibited Sexual Desire (ISD) was required for women and the Sexual desire inventory (SDI) was required for men. Non-probability sampling for convenience was implemented, descriptive statistics were applied, and the results are presented in a general way for the total population

Results: The prevalence of vitamin D deficiency was $63.55 \%$ in total, $34.57 \%$ in men and $28.97 \%$ in women. Vitamin D levels were lower in men than in women (34.57 vs. $41.36, \mathrm{p}<0.001$ ), with a higher percentage of deficiency $(21.49$ vs. $14.95 \%$ p <0.007) and of insufficiency (16.82 vs. $10.28 \%, \mathrm{p}<0.001)$.

Conclusion: The prevalence of vitamin D deficiency is considerably high in men and women with hypoactive sexual desire. It is necessary to quantify their vitamin D levels in order to establish the substitution as part of the treatment.

Key words: Vitamin D Deficiency; Physiological Sexual Dysfunctions,; Sexual Health; Vitamin D. 


\section{Introducción}

El deseo sexual hipoactivo (DSH) se define como una deficiencia (o ausencia) persistente o recurrente de fantasías sexuales y deseo de actividad sexual que causa angustia marcada o dificultad interpersonal, la cual no se explica de forma exclusiva por una condición médica o psiquiátrica o al uso de una sustancia (por ejemplo, medicamentos) (1). Alrededor del 32,97 \% al $39 \%$ de las mujeres reportan bajo deseo sexual, aunque solo un 8 $\%$ a $10 \%$ cumplen los criterios del diagnóstico primario (deseo bajo y sufrimiento asociado) (2,3). En los hombres la prevalencia oscila en el $6 \%$ (entre 18 a 24 años) y 41 $\%$ (entre 66 y 74 años) (4).

La etiología del DSH es multifactorial y compleja, por lo tanto, se puede atribuir a una serie de componentes biológicos, psicológicos, sociales y contextuales $(5,6)$. Es conocido el fundamental papel de las hormonas (andrógenos, progesterona, estrógenos, etc.) como factores involucrados en la modulación de la respuesta sexual $(5,7)$. El bajo nivel sérico de vitamina D se asocia con un funcionamiento sexual deficiente, y la severidad de la disfunción sexual dependerá de la gravedad de la deficiencia (8).

De los cuestionarios de autoevaluación, -los cuales han sido validados para evaluar el bajo deseo sexual en la mujer-, el Decreased Sexual Desire Screener (DSDS): (PDSI, Prueba del Deseo Sexual Inhibido), es útil para la consulta de cualquier profesional, tiene la ventaja de ser breve pudiéndose utilizar en mujeres antes y después de la menopausia (9). En hombres, el sexual desire inventory (SDI) es de utilidad (10).

El diagnóstico del DSH incluye investigar su aparición, duración, adaptación y evitación del comportamiento y el nivel de angustia. Se hace mandatario una evaluación detallada del historial médico y reproductivo, afecciones o comorbilidades (incluir trastornos endocrinos, neurológicos, cardiovasculares y psiquiátricos), así como el uso de medicamentos recetados o de venta libre. Se debe realizar un examen físico completo y específico para descartar cualquier otra causa de bajo deseo sexual $(5,11)$.

Es escasa la información donde se relacionen los bajos niveles séricos de vitamina $\mathrm{D}$ con bajo deseo sexual, sin embargo, es evidente su relación con trastornos sexuales en hombres (12) y mujeres (13). Es importante conocer si existe relación directa entre niveles bajos de vitamina D y la presencia de deseo sexual hipoactivo, a fin de planear y emprender acciones terapéuticas en la población con hipovitaminosis D. De este modo, la presente investigación tiene como objetivo principal determinar la prevalencia de déficit de vitamina $D$ en hombres y mujeres con deseo sexual hipoactivo en la ciudad de Armenia (Quindío), Colombia.

\section{Materiales y métodos}

Diseño y población: estudio observacional, descriptivo de corte transversal, realizado del 01 de mayo de 2011 al 30 de noviembre de 2017 en Armenia, capital del departamento del Quindío, Colombia. Se reclutó, de forma consecutiva, una muestra de 153 pacientes diagnosticados con deseo sexual hipoactivo (DSH), atendiendo a los criterios diagnósticos internacionales (1) en la consulta externa de una clínica privada de carácter universitario de referencia. El muestreo fue no probabilístico por conveniencia. Se incluyeron hombres y mujeres mayores de edad diagnosticados con deseo sexual hipoactivo (DSH). Se excluyeron analfabetas, gestantes, personas que presentaban enfermedad neoplásica, mujeres con menopausia quirúrgica, hombres prostatectomizados, personas con padecimientos metabólicos, trastornos mentales o déficit mental y los que no consintieron participar el estudio.

Procedimiento: Se seleccionaron las personas de la población de pacientes que asisten a la consulta de sexología del autor, respetando las normas de buena práctica clínica establecidas en la Declaración de Helsinki (14). La recolección de la información estuvo a cargo de las enfermeras, debidamente entrenadas en el proceso de captación de pacientes y diligenciamiento de formularios, y que son parte del equipo de investigación; las cuales encuestaban a las personas al momento de salir de la consulta con el especialista, previa verificación de los criterios de inclusión y exclusión. A las personas que cumplían con los criterios, se le informaban los objetivos de la investigación, así como el propósito que tenían los resultados, garantizándose la confidencialidad de la información. Igualmente, se le solicitaba la firma del consentimiento informado. Una vez firmado el consentimiento, las enfermeras encargadas de la recolección del instrumento estandarizado (el Decreased Sexual Desire Screener (DSDS) o Prueba del Deseo Sexual Inhibido (PDSI), para mujeres y el sexual desire inventory (SDI) para hombres), le daban instrucciones para el autodiligenciamiento del cuestionario, de manera individual y en un ambiente privado a cada uno de los participantes. En la Tabla 1 se observa el DSDS utilizado: 


\section{Tabla 1. Prueba del deseo sexual inhibido (PDSI) / Decreased Sexual Desire Screener (DSDS)}

1. ¿Considera que en el pasado su nivel de deseo o interés sexual era bueno y satisfactorio?

2. ¿Ha disminuido su deseo o interés sexual?

3. ¿Le molesta que su deseo o interés sexual haya disminuido?

4. ¿Le gustaría que aumentase su deseo o interés sexual?

5. Marque todos los factores que crea que pueden estar contribuyendo al actual descenso de su deseo o interés sexual:

A) Una operación, depresión, lesiones o cualquier otra afección.

B) Medicación, drogas o alcohol que usted está tomando actualmente.

C) Embarazo, parto reciente, síntomas de menopausia.

D) Otros problemas sexuales que pueda sufrir en la actualidad (dolor, disminución de la excitación o del orgasmo).

E) Los problemas sexuales de su pareja.

F) Insatisfacción con su relación o pareja.

G) Estrés o fatiga.

Sí No

Médico:

Si la paciente responde "NO" a cualquiera de las 4 primeras preguntas, entonces no cumple los criterios para ser diagnosticada del DSH adquirido.

Si la paciente responde "SI" " a todas las 4 primeras preguntas y su revisión confirma la respuesta "NO" en todos los apartados de la pregunta 5, entonces deberá ser diagnosticada del DSH adquirido.

Si la paciente responde "SI'" a todas las primeras 4 preguntas y "SI'" en cualquiera de los apartados de la quinta pregunta, entonces usted deberá decidir si las respuestas de la quinta pregunta indican un diagnostico primario diferente al de DSH adquirido. Las enfermedades concurrentes asociadas a la excitación o el orgasmo no deberían excluir la realización de un diagnostico simultaneo de DSH.

A partir de lo anterior, ¿sufre la mujer el trastorno del deseo sexual hipoactivo adquirido?

E1 PDSI es un instrumento utilizado para el diagnóstico del DSH adquirido, útil en mujeres antes y después de la menopausia (15); está conformado por 5 preguntas. Las 4 primeras preguntas evalúan si la mujer ha perdido su deseo sexual y si se siente afectada o molesta por ello, y la pregunta 5, de carácter compuesto, ayuda al médico a realizar el diagnóstico diferencial. Al cuestionario PDSI se le atribuye una precisión diagnóstica del 85,2 \% (sensibilidad y especificidad de 83,6 a 95,6 \% y 87,8 \%, respectivamente) $(15,16)$.

El sexual desire inventory (SDI) consta de 14 ítems que miden dos dimensiones: (A) deseo sexual diádico (ítems 1-9), y (B) deseo sexual solitario (ítems 10-13). El ítem 14 se refiere a la cantidad de tiempo que el individuo puede sentirse cómodo sin tener relaciones sexuales (10). 
A todos los participantes se les realizó laboratorios de ingreso: hemograma, niveles de vitamina D, BUN, creatinina, glucemia, TSH, prolactina, testosterona total y globulina fijadora de hormonas sexuales (SHBG: del inglés Sex Hormone Binding Globulin) para evaluar la existencia y posibles causas de DSH. Cada uno de los resultados de cada paciente, así como los hallazgos del PDSI y del SDI fue registrado en un formato especial diseñado por los investigadores.

Variables medidas: Sociodemográficas (edad, raza, escolaridad, estrato socioeconómico, estado civil, ocupación, convivencia, condición espiritual o religiosa, área de residencia, edad de la pareja); variables de salud sexual y reproductiva (paridad, edad de la menopausia, tabaquismo, ingesta de alcohol, uso de anticoncepción, antecedente personal de depresión o de disfunción sexual, uso de terapia hormonal de reemplazo); variables de comportamiento sexual (orientación sexual, edad de la primera relación sexual, práctica de la masturbación, frecuencia promedio de relaciones coitales mensuales, satisfacción con la vida sexual, número de parejas sexuales, tiempo de convivencia en pareja, antecedente de abuso sexual o violencia sexual en el matrimonio, pareja con disfunción sexual e infidelidad). Se indagaron además las preguntas de los dominios de la encuesta PDSI y SDI.

Análisis estadístico: Los cálculos estadísticos se realizaron con el programa EPIDAT 3.1. Las variables cualitativas se expresaron como frecuencias absolutas y relativas (porcentajes), y las cuantitativas como media y desviación estándar (DE). Los resultados se exponen de manera agrupada para el total de la población. Se presenta la prevalencia de disfunción sexual global y por sexos.

Aspectos éticos: se cumplieron los requisitos para la investigación en humanos según la Declaración de Helsinki y la Resolución 8430 de 1993 del Ministerio de Salud de Colombia.

\section{Resultados}

De un total de 153 personas asistentes a consulta por deseo sexual hipoactivo, se incluyeron 134 (87,58 \%), de las cuales siete $(5,22 \%)$ se negaron a participar. De los 127 restantes que cumplieron los criterios de selección, un total de $11(8,66 \%)$ no se realizaron el examen de la vitamina $\mathrm{D}$, otras $9(8,41 \%)$ no volvieron a la revisión de los estudios, por lo cual fueron excludos.

Se incluyeron un total de 107 pacientes para la investigación. Las mujeres predominaron con 64 participantes $(59,81 \%)$. La edad media de la población global fue de 43,56 \pm 9,31 años (límites entre 18 y 63 años); predominaron hispánicos, estrato alto, residentes del área urbana, casados, católicos, profesionales y heterosexuales. La obesidad estuvo presente en el 30,18 $\%$ de los hombres y $23,43 \%$ de las mujeres. El 16,27 \% de los hombres y $15,62 \%$ de las mujeres eran fumadores crónicos. La edad de la pareja fue 38,47 $\pm 8,25$ años en los hombres (límites entre 28 y 51 años), mientras en las mujeres la edad de la pareja fue de 50,68 \pm 9,71 años (límites entre 47 y 63 años). La edad de la menopausia fue de 49,7 $\pm 5,8$ años. No se observaron, entre los sexos, diferencias estadísticamente significativas $(p>0,05)$, excepto en la talla $(p=0,024)$ y el peso $(p=0,027)$, y por ende en el IMC. A continuación, se relacionan los datos en la Tabla 2:

\begin{tabular}{|c|c|c|c|}
\hline & $\begin{array}{c}\text { Hombres } \\
n=43\end{array}$ & $\begin{array}{c}\text { Mujeres } \\
n=64\end{array}$ & $\mathrm{p}$ \\
\hline Edad, $\mathrm{X} \pm \mathrm{DE}$ años & $46,35 \pm 5,24$ & $42,64 \pm 6,15$ & 0,645 \\
\hline Peso, $\mathrm{X} \pm \mathrm{DE}$ & $79,41 \pm 8,32$ & $65,37 \pm 5,62$ & 0,027 \\
\hline Talla, $\mathrm{X} \pm \mathrm{DE}$ & $1,68 \pm 0,94$ & $1,57 \pm 0,89$ & 0,024 \\
\hline $\mathrm{IMC}, \mathrm{X} \pm \mathrm{DE}$ & $28,92 \pm 4,36$ & $25,23 \pm 3,71$ & 0,375 \\
\hline \multicolumn{4}{|l|}{ Raza } \\
\hline Hispánica, n (\%) & $27(62,79)$ & $36(56,25)$ & 0,483 \\
\hline Afrocolombiana, n (\%) & $12(27,9)$ & $18(28,12)$ & 0,081 \\
\hline Indígena, n (\%) & $4(9,3)$ & $10(15,62)$ & 0,069 \\
\hline \multicolumn{4}{|l|}{ Estado civil } \\
\hline Casadas, n (\%) & $24(55,81)$ & $27(42,18)$ & 0,351 \\
\hline Unión libre, n (\%) & $14(32,55)$ & $24(37,5)$ & 0,414 \\
\hline Pareja estable, n (\%) & $5(11,62)$ & $13(20,31)$ & 0,093 \\
\hline \multicolumn{4}{|l|}{ Estrato socio-económico } \\
\hline Alto, n (\%) & $30(69,76)$ & $39(60,93)$ & 0,729 \\
\hline Medio, n (\%) & $12(27,9)$ & $21(32,81)$ & 0,138 \\
\hline Bajo, n (\%) & $1(2,32)$ & $4(6,25)$ & 0,174 \\
\hline \multicolumn{4}{|l|}{ Ocupación } \\
\hline Trabajador independiente, $\mathrm{n}(\%)$ & $15(34,88)$ & $18(28,12)$ & 0,723 \\
\hline Empleadas, n (\%) & $21(48,83)$ & $33(51,56)$ & 0,264 \\
\hline Pensionadas, n (\%) & $7(16,27)$ & $13(20,31)$ & 0,159 \\
\hline \multicolumn{4}{|l|}{ Escolaridad } \\
\hline Secundaria, n (\%) & $7(16,27)$ & $10(15,62)$ & 0,108 \\
\hline Técnica, n (\%) & $12(27,9)$ & $18(28,12)$ & 0,297 \\
\hline Universitarios, n (\%) & $24(55,81)$ & $36(56,25)$ & 0,348 \\
\hline \multicolumn{4}{|l|}{ Origen } \\
\hline Urbana, n (\%) & $28(65,11)$ & $46(71,87)$ & 0,165 \\
\hline Rural, n (\%) & $15(34,88)$ & $18(28,12)$ & 0,285 \\
\hline Heterosexual, n (\%) & $39(90,69)$ & $57(89,06)$ & 0,177 \\
\hline Homosexual / Bisexual, n (\%) & $4(9,3)$ & $7(10,93)$ & 0,168 \\
\hline Antecedente personal de depresión, n (\%) & $5(11,62)$ & $8(12,5)$ & 0,204 \\
\hline Antecedente de disfunción sexual, n (\%) & $9(20,93)$ & $18(28,12)$ & 0,075 \\
\hline Antecedente de abuso sexual, n (\%) & $6(13,95)$ & $9(14,06)$ & 0,102 \\
\hline Tabaquismo, n (\%) & $7(16,27)$ & $10(15,62)$ & 0,168 \\
\hline Ingesta de alcohol, n (\%) & $12(27,9)$ & $15(23,43)$ & 0,147 \\
\hline \multicolumn{4}{|l|}{ Condición espiritual } \\
\hline Católica, n (\%) & $36(83,72)$ & $51(79,28)$ & 0,279 \\
\hline Testigos de Jehová, n (\%) & $4(9,3)$ & $6(9,37)$ & 0,108 \\
\hline Otras, n (\%) & $3(6,97)$ & $7(10,93)$ & 0,609 \\
\hline
\end{tabular}


La edad promedio del inicio de la actividad sexual en los hombres fue de 15,42 $\pm 2,56$ años y la de las mujeres de $16,72 \pm 2,38$ años. La práctica sexual más frecuente entre las mujeres fue el coito vaginal $(84,37 \%)$, mientras que la masturbación fue considerada una práctica poco común en ellas $7,81 \%$, mientras en los hombres la masturbación alcanzó el 27,9\%.

El 82,24 \% de los pacientes afirmó dificultades de pareja por su trastorno sexual, acompañándose de baja autoestima en el 79,43\%. Veintisiete $(42,18 \%)$ de las mujeres afirmaron haberse separado de sus parejas por este conflicto, de las cuales $18(28,12 \%)$ describieron haber sido abusadas por sus parejas.

Se encontró una mediana de 2 hijos en la paridad (un rango entre 0 y 6 hijos), hubo un 76,56\% de antecedente de episiotomía. El método anticonceptivo predominante en las mujeres fue el hormonal $(81,25 \%)$, mientras que la terapia hormonal de reemplazo se encontró en el 30,76 $\%$ de las mujeres mayores de 50 años. El 11,32 \% de los hombres se había realizado la vasectomía.

El número de parejas sexuales reportado entre los hombres arrojó una mediana de 9 (rango entre 1 y 15), en las mujeres 5 (rango entre 1 y 9). El 45,79\% de los participantes en el estudio manifestó más de 15 años de tiempo de convivencia en pareja. El 79,68 \% de las mujeres y el 43,39\% de los hombres manifestaron que la pareja presentaba alguna disfunción sexual. El 49,05 $\%$ de los hombres y el $14,06 \%$ de las mujeres afirmaron haber sido infiel, por lo menos en una ocasión. El 76,56 $\%$ de las mujeres y el 15,09\% de los hombres refirieron que la pareja le había sido infiel.

A la pregunta ¿cuántas veces tuvo relaciones sexuales el mes pasado? ( $\square$ período definido como el lapso de los treinta días anteriores $\square$ ), el 79,24\% de los hombres y el $39,06 \%$ de las mujeres informó tener relaciones cuatro veces por mes (rango entre 1 y 6). El 76,63\% de la población total, informó haberse automedicado para tratar su disfunción, el 18,69\% había consultado previamente en una ocasión por la disfunción sexual. En el grupo de hombres el 69,81\% había ingerido sildenafil por lo menos en una ocasión, mientras que el 16,98\% lo hacía de forma regular (1 a 3 veces por semana).

La prevalencia de DSH asociada a malestar o sufrimiento fue del $50,94 \%$ en los hombres y del $26,56 \%$ en las mujeres. El 90,56 \% de los hombres y el 78,12\% de las mujeres manifestaron no estar satisfechos con su vida sexual. El tiempo de evolución de la disfunción sexual mostró un recorrido de 1 a 12 años (promedio: 13,24 \pm 5,79 años).

En la población total de pacientes, $68(63,55 \%)$ de los 107 participantes presentaban déficit de vitamina $D$ en el momento del estudio (39 deficiencia, 29 insuficiencia), de los cuales $37(34,57 \%)$ eran hombres y $31(28,97$ $\%$ ) eran mujeres. En la comparación entre los sexos, no se encontraron diferencias en la distribución por edad, origen, raza u orientación sexual. Sin embargo, en la población total de participantes, el promedio de los niveles de vitamina $\mathrm{D}$ fue inferior en los hombres respecto a las mujeres, $(34,57$ vs. 41,36 ; $p<0,001)$, con mayor porcentaje de deficiencia $(21,49$ vs. $14,95 \%$ $\mathrm{p}<0,007)$ y de insuficiencia $(16,82$ vs. $10,28 \%, \mathrm{p}<0,001)$.

Las determinaciones de los niveles séricos de glucemia reportaron una media de 102,18 $\pm 8,04$ (límites 86,19 a $198,27 \mathrm{mg} / \mathrm{dL}$ ), donde el $18,6 \%$ de los hombres y el 12,5 $\%$ de las mujeres reportaron una glucemia $>126 \mathrm{mg} / \mathrm{dL}$, compatible con diabetes; la TSH arrojó una media de $3,87 \pm 1,53$ (límites 0,81 a 14,28 mU/L), el 6,97\% de los hombres y el 7,81\% de las mujeres reportaron una TSH $>10 \mathrm{mU} / \mathrm{L}$, compatible con hipotiroidismo; la prolactina mostró una media de 17,4 \pm 5,31 (límites 8,13 a 23,61 ng/ dL), el 4,65\% de los hombres y el 4,68\% de las mujeres reportaron una prolactina $>35 \mathrm{ng} / \mathrm{mL}$ diagnóstica de hiperprolactinemia, sin embargo, la testosterona total se encontraba en limites inferiores, tanto en hombres como mujeres, reportando en los hombres una media de 384,27 $\pm 76,83$ (límites 195,48 a 716,43 ng/dL), mientras en las mujeres la media fue de 28,47 $\pm 11,58$ (límites 15,24 a $45,21 \mathrm{ng} / \mathrm{dL}$ ). Se observó una disminución significativa de la SHBG, tanto en hombres como mujeres, arrojando en los hombres una media de 19,47 nmol/L $\pm 4,83$ (límites 16,29 a 58,26 nmol/L), mientras en las mujeres la media fue de $36,87 \mathrm{nmol} / \mathrm{L} \pm 7,35$ (límites 15,81 a $71,34 \mathrm{nmol} / \mathrm{L})$.

En los hombres con deficiencia de vitamina D, la testosterona total arrojó una media de 273,14 $\pm 65,91$ (límites 137,76 a 327,24 ng/dL), y la SHBG reportó una media de $16,83 \mathrm{nmol} / \mathrm{L} \pm 4,71$ (límites 16,89 a 58,26 $\mathrm{nmol} / \mathrm{L}$ ), mientras en los que presentaron insuficiencia fue de 210,87 \pm 49,26 (límites 104,91 a 293,37 ng/dL) y la SHBG registró una media de $16,26 \mathrm{nmol} / \mathrm{L} \pm 4,05$ (límites 16,29 a 49,71 nmol/L); por su parte las mujeres con deficiencia de vitamina $\mathrm{D}$ presentaron una media de $23,52 \pm 6,75$ (límites 15,81 a $27,39 \mathrm{ng} / \mathrm{dL}$ ) y la SHBG una media de $32,52 \mathrm{nmol} / \mathrm{L} \pm 6,42$ (límites 19,74 a 71,34 $\mathrm{nmol} / \mathrm{L}$ ), mientras aquellas con insuficiencia reportaron $18,57 \pm 4,65$ (límites 10,17 a $23,79 \mathrm{ng} / \mathrm{dL}$ ) y la SHBG una media de $30,48 \mathrm{nmol} / \mathrm{L} \pm 5,58$ (límites 15,81 a 62,46 $\mathrm{nmol} / \mathrm{L})$.

\section{Discusión}

Durante los seis años de seguimiento, se obtuvo una prevalencia de déficit de vitamina $\mathrm{D}$, en hombres y mujeres con deseo sexual hipoactivo del $63,55 \%$; de los cuales el $34,57 \%$ eran hombres y el $28,97 \%$ eran mujeres. El promedio de los niveles de vitamina $\mathrm{D}$ fueron 
inferiores en los hombres respecto a las mujeres, $(34,57$ vs. 41,$36 ; p<0,001)$, con mayor porcentaje de deficiencia $(21,49$ vs. $14,95 \% \mathrm{p}<0,007)$ y de insuficiencia $(16,82$ vs. $10,28 \%, p<0,001)$; coincidiendo con niveles bajos de testosterona total (media de $273,14 \pm 65,91$ y $210,87 \pm$ 49,26, para deficiencia e insuficiencia, respectivamente) y bajos niveles séricos de SHBG 16,83 $\pm 4,71 \mathrm{nmol} / \mathrm{L}$ y $16,26 \pm 4,05 \mathrm{nmol} / \mathrm{L}$, respectivamente.

Los estudios actuales evidencian un significativo papel de la vitamina $\mathrm{D}$ en la regulación de la función sexual, sugiriendo que los niveles bajos de vitamina D están asociados con la aparición de trastornos sexuales (12). Nuestros resultados son parecidos a lo reportado en el estudio realizado por Farag et al., que manifiesta una prevalencia ponderada de deficiencia de vitamina D y trastorno sexual del $30 \%$ (17), pero otros autores muestran una prevalencia de $45,9 \%$ y $81 \%$, realizados en poblaciones similares y con comorbilidades parecidas $(12,18)$; otros estudios hechos en poblaciones de mujeres mostraron la relación entre la deficiencia de vitamina D y la presencia de trastornos sexuales, tal como lo describe Canat et al., quienes de acuerdo al puntaje total del Índice de Función Sexual Femenino (IFSF) encontró correlación entre una baja puntación y bajos viles de vitamina D (19).

En los hombres con niveles vitamina $\mathrm{D}$ por debajo de 20 $\mathrm{ng} / \mathrm{ml}$ se han reportado formas más severas de trastornos sexuales, especialmente relacionados con disfunción eréctil; mientras que, en las mujeres, dicho déficit afecta, predominantemente, el deseo sexual (13), tal como lo hemos encontrado en nuestro estudio. De esto se desprende que la vitamina D influye en la capacidad de regular el ciclo de respuesta sexual, tanto en hombres como en mujeres. Lo cual ha sido correlacionado por Canguven et al., quienes reportaron la existencia de una asociación entre la mejora inducida por la vitamina $\mathrm{D}$ en los trastornos sexuales, paralela a un aumento en los niveles de testosterona (20), resultados reportados también por el estudio de Anic et al. (21); sin embargo, no se descarta que los trastornos sexuales presentes en personas con déficit de vitamina $\mathrm{D}$, sean resultado de una neuropatía, cuyo riesgo se correlacionó con los niveles de vitamina D en el estudio de Jung CH et al. (22).

A diferencia del estudio de Gozdzik et al. que evaluó la influencia de la variación estacional en los niveles de vitamina D (23), nosotros en esta investigación no lo consideramos, pero aceptamos la posibilidad de que el funcionamiento sexual asociado con niveles bajos de vitamina $\mathrm{D}$ puedan ser diferentes si el estudio se realizara teniendo en cuenta las condiciones climáticas, además de destacar la correlación existente entre el déficit de vitamina $\mathrm{D}$ y niveles bajos de testosterona total, tal como ha sido descrito por diferentes autores $(12,13,17,19)$.
La presente investigación tiene como fortalezas lo representativo de la muestra, la evaluación de los niveles de vitamina $\mathrm{D}$ como factor asociado a deseo sexual hipoactivo tanto en hombres como en mujeres. Entre las limitaciones se destacan el número de personas excluidas, el hecho de haberse realizado en la consulta del autor no pudiéndose extrapolar a toda la región del Eje Cafetero; y que además no se evaluaron los niveles séricos de la hormona paratiroidea (PTH) para correlacionar sus niveles respecto al déficit de vitamina $\mathrm{D}$, variables que pueden incidir en el incremento en la prevalencia del deseo sexual hipoactivo.

\section{Conclusiones}

La prevalencia del déficit de vitamina $\mathrm{D}$ en personas con deseo sexual hipoactivo fue del 63,55\%. El déficit de vitamina $\mathrm{D}$ se asocia con deseo sexual hipoactivo en hombres y mujeres. Estudios posteriores, donde se incluya un mayor tamaño de la muestra, se hacen necesarios para entender la influencia del déficit de vitamina D en la aparición del deseo sexual hipoactivo.

\section{Agradecimientos}

Les agradecemos a las personas que nos permitieron llevar a cabo el proceso de esta novedosa investigación. Al doctor Mauricio Delgado García por su incondicional apoyo, tanto por la colaboración como por la efectividad de su orientación académica, sin cuyo apoyo no hubiese sido posible lograr el resultado final.

\section{Financiación}

La presente investigación no cuenta con la financiación ni patrocinio alguno.

Conflicto de intereses: ninguno declarado.

\section{Bibliografía:}

1. American Psychiatric Association. Diagnostic and statistical manual of mental disorders. 4th ed, text rev. Washington,DC:American PsychiatricAssociation; 2000.

2. Espitia-De La Hoz FJ. Prevalence and characterisation of sexual dysfunctions in women, in 12 Colombian cities, 2009-2016. Rev Colomb Obstet Ginecol 2018; 69(1):9-21. doi:10.18597/rcog.3035

3. Shifren JL, Monz BU, Russo PA, et al. Sexual problems and distress in United States women: prevalence and correlates. Obstet Gynecol 2008; 112:970-978. doi: 10.1097/AOG.0b013e3181898cdb

4. Corona G, Trípodi F, Reisman J, Maggi M. Male hypoactive sexual desire disorder. In: The EFS and ESSM Syllabus of Clinical Sexology. 
Amsterdam: Medix Publishers; 2016. p. 532-55.

5. Espitia-De La Hoz, FJ. Fisiopatología del trastorno del deseo sexual en el climaterio. Rev Méd Risaralda. 2018; 23(1): 54-59.

6. Clayton AH. The pathophysiology of hypoactive sexual desire disorder in women. Int $\mathrm{J}$ Gynaecol Obstet 2010; 110:7-11. doi: 10.1016/j.ijgo.2010.02.014

7. Shifren JL, Braunstein GD, Simon JA, Casson PR, Buster JE, Redmond GP, et al. Transdermal testosterone treatment in women with impaired sexual function after oophorectomy. N Engl J Med 2000; 343(10): 682-8. doi: 10.1056/NEJM200009073431002

8. Colonese F, Laganà AS, Colonese E, Sofo V, Salmeri FM, Granese R, et al. The pleiotropic effects of vitamin D in gynaecological and obstetric diseases: an overview on a hot topic. Biomed Res Int. 2015; 2015:986281. doi: 10.1155/2015/986281

9.Clayton AH, Goldfischer ER, Goldstein I, DeRogatis L, Lewis-D'Agostino D, Pyke R. Validation of the Decreased Sexual Desire Screener (DSDS): A brief diagnostic instrument for generalized acquired female Hypoactive Sexual Desire Disorder (HSDD). J Sex Med. 2009; 6:730-8. doi: 10.1111/j.1743-6109.2008.01153.x

10. Spector IP, Fremeth SM. Sexual behaviors and attitudes of geriatric residents in long-term care facilities. Journal of Sex \& Martial Therapy. 1996; 22:235-246. doi:10.1080/00926239608404402

11. Kingsberg SA, Woodard T. Female sexual dysfunction: focus on low desire. Obstet Gynecol 2015; 125:477-486. doi: 10.1097/AOG.0000000000000620

12. Barassi A, Pezzilli R, Colpi GM, Corsi Romanelli MM, Melzi d'Eril GV. Vitamin D and erectile dysfunction. J Sex Med. 2014;11(11):2792-800. doi: $10.1111 /$ jsm. 12661

13. KrysiakR, GilowskaM,OkopieńB.Sexualfunctionand depressive symptoms in young women with low vitamin D status: a pilot study. Eur J Obstet Gynecol Reprod Biol. 2016; 204: 108-112, doi: 10.1016/j.ejogrb.2016.08.001

14. De Roy P. Helsinki and the Declaration of Helsinki. World Med J. 2004; 50:9---11.21.

15.Goldfischer ER, Clayton AH, Goldstein I, LewisD'Agostino D, Pyke R: Decreased Sexual Desire Screener (DSDS) for diagnosis of hypoactive sexual desire disorder in women. Obstet. Gynecol. 2008; 111:109.
16. Nappi RE, Dean J, Hebert A, Pyke R: Decreased Sexual Desire Screener (DSDS) for diagnosis of Hypoactive Sexual Desire Disorder (HSDD) in European women. J. Sex. Med. 2009;6 Suppl. 2:46.

17. Farag YM, Guallar E, Zhao D, Kalyani RR., Blaha MJ, Feldman DI, et al. Vitamin D deficiency is independently associated with greater prevalence of erectile dysfunction: The National Health and Nutrition Examination Survey (NHANES) 2001-2004. Atherosclerosis. 2016; 252:61-67. doi: 10.1016/j.atherosclerosis.2016.07.921

18. Bellastella G, Maiorino MI, Olita L, Capuano A, Rafaniello C, Giugliano D, et al. Vitamin D deficiency in type 2 diabetic patients with hypogonadism. J Sex Med. 2014;11(2):536-42. doi: $\quad 10.1111 / \mathrm{jsm} .12384$

19. Canat M, Canat L, Öztürk FY, Eroğlu H, Atalay HA, Altuntaş Y. Vitamin D3 deficiency is associated with female sexual dysfunction in premenopausal women. Int Urol Nephrol. 2016 Nov;48(11):1789-1795.

20. Canguven O, Talib RA, El Ansari W, Yassin DJ, Al Naimi A. Vitamin D treatment improves levels of sexual hormones, metabolic parameters and erectile function in middle-aged vitamin D deficient men. Aging Male. 2017; 11:1-8.

21. Anic GM, Albanes D, Rohrmann S, Kanarek $\mathrm{N}$, Nelson WG, Bradwin $\mathrm{G}$, et al. Association between serum 25-hydroxyvitamin $\mathrm{D}$ and serum sex steroid hormones among men in NHANES. Clin Endocrinol (Oxf). 2016; 85:258-66.

22. Jung CH, Jung SH, Kim KJ, Kim BY, Kim CG, Kang $\mathrm{SK}$, et al. The relationship between vitamin $\mathrm{D}$ status and cardiac autonomic neuropathy in patients with type 2 diabetes mellitus. Diab Vasc Dis Res. 2015; 12:342-51.

23. Gozdzik A, Barta JL, Weir A, Cole DE, Vieth R, Whiting SJ, et al. Serum 25-hydroxyvitamin D concentrations fluctuate seasonally in young adults of diverse ancestry living in Toronto. J Nutr. 2010; 140:2213-20. 